\title{
Tight Information-Theoretic Lower Bounds for Welfare Maximization in Combinatorial Auctions
}

\author{
Vahab Mirrokni \\ Theory Group, Microsoft \\ Research \\ Redmond, WA 98052 \\ mirrokni@theory.csail.mit.edu
}

\author{
Michael Schapira ${ }^{*}$ \\ School of Engineering and \\ Computer Science \\ Hebrew University of \\ Jerusalem, Israel \\ mikesch@cs.huji.ac.il
}

\author{
Jan Vondrák \\ Dept. of Mathematics \\ Princeton University \\ Princeton, NJ 08544 \\ jvondrak@gmail.com
}

\begin{abstract}
We provide tight information-theoretic lower bounds for the welfare maximization problem in combinatorial auctions. In this problem, the goal is to partition $m$ items among $k$ bidders in a way that maximizes the sum of bidders' values for their allocated items. Bidders have complex preferences over items expressed by valuation functions that assign values to all subsets of items.

We study the "black box" setting in which the auctioneer has oracle access to the valuation functions of the bidders. In particular, we explore the well-known value query model in which the permitted query to a valuation function is in the form of a subset of items, and the reply is the value assigned to that subset of items by the valuation function.

We consider different classes of valuation functions: submodular, subadditive, and superadditive. For these classes, it has been shown that one can achieve approximation ratios of $1-\frac{1}{e}, \frac{1}{\sqrt{m}}$, and $\frac{\sqrt{\log m}}{m}$, respectively, via a polynomial (in $k$ and $m$ ) number of value queries. We prove that these approximation factors are essentially the best possible: For any fixed $\epsilon>0$, a $(1-1 / e+\epsilon)$-approximation for submodular valuations or an $\frac{1}{m^{1 / 2-\epsilon}}$-approximation for subadditive valuations would require exponentially many value queries, and a $\frac{\log ^{1+\epsilon} m}{m}$-approximation for superadditive valuations would require a superpolynomial number of value queries.
\end{abstract}

\section{Categories and Subject Descriptors}

F.2 [Theory of Computation]: ANALYSIS OF ALGORITHMS AND PROBLEM COMPLEXITY

\section{General Terms}

Algorithms, Economics, Theory

${ }^{*}$ Supported by grants from the Israel Science Foundation.

Permission to make digital or hard copies of all or part of this work for personal or classroom use is granted without fee provided that copies are not made or distributed for profit or commercial advantage and that copies bear this notice and the full citation on the first page. To copy otherwise, to republish, to post on servers or to redistribute to lists, requires prior specific permission and/or a fee.

EC'08, July 8-12, 2008, Chicago, Illinois, USA.

Copyright 2008 ACM 978-1-60558-169-9/08/07 ...\$5.00.

\section{Keywords}

Approximation Algorithms, Combinatorial Auctions

\section{INTRODUCTION}

Combinatorial auctions are a central research area at the intersection of economics, game theory, and computer science. The welfare maximization problem in combinatorial auctions is an abstraction of many computational and economic resource-allocation problems. In this problem, an auctioneer sells a set $M$ of $m$ items to a set of $k$ bidders. The value of bidder $i$ for any subset (bundle) of items is given by a valuation set function $v_{i}: 2^{M} \rightarrow \mathbb{R}_{+}$, where $v_{i}(S)$ represents $i$ 's maximum willingness to pay for the bundle $S$. The two standard assumptions on each $v_{i}$ are that if $S \subseteq T$ then $v_{i}(S) \leq v_{i}(T)$ (monotonicity), and that $v_{i}(\emptyset)=0$ (normalization). The objective is to partition $M$ into $k$ disjoint subsets $S_{1}, S_{2}, \ldots, S_{k}$ in a way that maximizes the expression $\sum_{1 \leq i \leq k} v_{i}\left(S_{i}\right)$ (i.e., the social welfare).

Algorithms for maximizing welfare in combinatorial auctions are required to be polynomial in the natural parameters of the problem, $m$ and $k$. However, since the "input" (the valuation functions) is of exponential size one must specify how it can be accessed. Most works in this field have taken a "black box" approach in which bidders' valuation functions are accessed via oracles that can answer specific type of queries. Three types of queries have been considered $[1,2,5]$ :

- Value queries: The query to a valuation function $v_{i}$ is in the form of a bundle $S \subseteq M$, and the response is $v_{i}(S)$.

- Demand queries: The query to a valuation function $v_{i}$ is in the form of a price vector $p=\left(p_{1}, \ldots, p_{m}\right)$ and the response is the bundle $T$ most demanded by $v_{i}$ given these prices. That is, $T=\operatorname{argmax}_{S \subseteq M} v_{i}(S)-$ $\sum_{j \in S} p_{j}$

- General queries: We allow any type of query (to each valuation function alone). This model captures the communication complexity (between the bidders) of the problem. Due to its strength it is mostly interesting for proving lower bounds.

Value queries are strictly less powerful than demand queries, which, in turn, are strictly less powerful than general queries [1, $2, ?]$. In this paper we focus our attention on the value query model. 
It has been shown that computing an optimal solution for social-welfare maximization requires an exponential number of queries even in the general queries model [13]. Hence, we are interested in the approximability of this problem. For the general case, the approximability of the problem is wellunderstood in all models $[1,13]$. Researchers have also studied the approximability of the problem for restricted classes of valuation functions. Two families of such classes, that have natural economic interpretations $[11,5]$, have been considered: Subadditive functions, and superadditive functions. A set function $f: 2^{M} \rightarrow \mathbb{R}_{+}$is subadditive iff for any two sets $S$ and $T, f(S)+f(T) \geq f(S \cup T)$, and is superadditive iff for any two disjoint sets $S$ and $T, f(S)+f(T) \leq f(S \cup T)$. An important special case of subadditive functions are $s u b$ modular functions. A set function $f$ is submodular iff for any two sets $S$ and $T, f(S)+f(T) \geq f(S \cup T)+f(S \cap T)$. Submodular functions (that are monotone and normalized) are also reasonable to consider from an economic perspective as they characterize functions with decreasing marginal utilities [11].

We present tight information-theoretic lower bounds for submodular, subadditive, and superadditive valuation functions in the value query model. We prove the following theorems:

Theorem: For any fixed $\epsilon>0$, achieving an approximation ratio of $1-\frac{1}{e}+\epsilon$ for welfare-maximization with submodular functions requires an exponential number of value queries.

This matches the $\left(1-\frac{1}{e}\right)$-approximation (achieved with a polynomial number of value queries) recently shown by Vondrák [15], who improved over the $\frac{1}{2}$-approximation shown by Lehmann et al. [11]. (We note that this problem can be formalized as the problem of maximizing a submodular function subject to a matroid constraint. Hence, the greedy algorithm developed by Fisher et al. [14] provides a $\frac{1}{2}$-approximation for this problem). The only previously known information-theoretic lower bound for this problem was $1-O\left(\frac{1}{m}\right)$ (see [13], in the general queries model. Our lower bound strengthens the $1-\frac{1}{e}+\epsilon$ lower bound dependent on $P \neq N P$ proven by Khot et al. [10]. We stress that our lower bound is independent of any computational complexity assumptions and holds even for algorithms of unbounded computational power, that are bounded only in terms of the number of value queries they can make. Also, we remark that the same inapproximability result does not hold in stronger query models - it is known that $(1-1 / e+\epsilon)$ approximation is possible with polynomially many demand queries [7].

Theorem: For any fixed $\epsilon>0$, achieving an approximation ratio of $\frac{1}{m^{\frac{1}{2}-\epsilon}}$ for welfare-maximization with subadditive functions requires an exponential number of value queries.

This matches the upper bound of $\frac{1}{\sqrt{m}}$ presented by Dobzinski et al. [5] (achieved using a polynomial number of value queries). The previously known lower bound in the value query model was $\frac{1}{m^{\frac{1}{4}}}$ [4]. In fact, our lower bound holds even for the more restricted subclass of fractionally subadditive valuations [6], introduced in [12] under the name of XOS.

Theorem: For any fixed $\epsilon>0$, achieving an approximation ratio of $\frac{\log ^{1+\epsilon} m}{m}$ for welfare-maximization with superadditive functions requires a super-polynomial number of value queries.

This nearly matches the upper bound of $\frac{\sqrt{\log m}}{m}$ presented by Holzman et al. [9] (achieved via a polynomial number of value queries). A similar lower bound was known for general valuation functions $[1,2]$. We extend this lower bound to the restricted class of superadditive functions. In fact, the lower bound holds for a superadditive analogue of fractionally subadditive functions (that is strictly contained in the class of superadditive valuation functions).

\section{VALUE-QUERY COMPLEXITY OF SUB- MODULAR WELFARE MAXIMIZATION}

In this section, we construct an example showing that it is impossible to achieve an approximation factor better than $1-1 / e$ for submodular utility functions in the value oracle model. We consider algorithms whose running time is potentially unbounded, we only count the number of value queries posed by the algorithm. More precisely, we prove the following.

Theorem 2.1. For any fixed $\beta>0$ and $k \geq 2$, any (possibly randomized) $\left(1-(1-1 / k)^{k}+\beta\right)$-approximation algorithm for $m$ items and $k$ players with submodular valuation functions requires $e^{\Omega(m)}$ value queries, otherwise it fails with high probability.

Since $1-(1-1 / k)^{k}$ is arbitrarily close to $1-1 / e$ for large enough $k$, this implies the following.

Corollary 2.2. For any fixed $\epsilon>0$, there is no (1$1 / e+\epsilon)$-approximation for an arbitrary number of players, using a subexponential number of queries.

We note that our examples use the same submodular valuation function for all players, just like in the NP-hardness result of [10]. Thus the problem is hard to approximate even in the special case where all utility functions are equal. Our construction is different from [10], however. While the hardness reduction of [10] uses explicit coverage-type submodular functions, our valuation functions are not exactly of the coverage type. Our construction is inspired by a lower bound developed by Feige et al. [8] for the problem of maximizing non-monotone submodular functions.

\section{Overview of the Proof.}

Consider a $k$-uniform hypergraph $H=(X, E)$ and a function $f: 2^{X} \rightarrow \mathbb{R}_{+}$where $f(S)$ is the number of hyperedges incident with the set of vertices $S$. This is a coverage-type submodular function. The idea is that it is hard to distinguish instances where $H$ is a complete $k$-partite $k$-uniform hypergraph (and allocating one part $X_{i}$ to each player results in a "perfect solution"), and instances where $H$ is a complete $k$-uniform hypergraph (and then there is no "perfect solution"). Since vertices of the hypergraph could be labeled arbitrarily on the input, it's hard for any algorithm to find a set of vertices significantly overlapping with any $X_{i}$ and hence it cannot distinguish these two cases.

In order to make the example work, we have to modify the coverage-type functions slightly. We consider a ground set $X$ partitioned into $X_{1} \cup X_{2} \cup \ldots \cup X_{k}$. The functions 
$f(S)$ that we define depend only on the fractions of $X_{i}$ that $S$ contains: $x_{i}=\left|S \cap X_{i}\right| /\left|X_{i}\right|$. To simplify the notation, we work with continuous functions $\tilde{f}\left(x_{1}, \ldots, x_{k}\right)$. The following lemma states the properties that we need $\tilde{f}\left(x_{1}, \ldots, x_{k}\right)$ to satisfy.

Lemma 2.3. Let $X=X_{1} \cup X_{2} \cup \ldots \cup X_{k}$ as above and let $\tilde{f}:[0,1]^{k} \rightarrow \mathbb{R}$ be a function with continuous first partial derivatives, and second partial derivatives almost everywhere. Define a discrete function $f: X \rightarrow \mathbb{R}$ so that

$$
f(S)=\tilde{f}\left(\frac{\left|S \cap X_{1}\right|}{\left|X_{1}\right|}, \ldots, \frac{\left|S \cap X_{k}\right|}{\left|X_{k}\right|}\right) .
$$

1. If $\frac{\partial \tilde{f}}{\partial x_{i}} \geq 0$ everywhere for each $i$, then the function $f$ is monotone.

2. If $\frac{\partial^{2} \tilde{f}}{\partial x_{i} \partial x_{j}} \leq 0$ almost everywhere ${ }^{1}$ for any $i, j$, then the function $f$ is submodular.

Proof. For monotonicity, it's sufficient to observe that if $\frac{\partial f}{\partial x_{i}} \geq 0$, then $\tilde{f}$ is non-decreasing in each coordinate. Hence, adding elements cannot decrease the value of $f$.

For the submodularity condition, fix an element in $a \in X_{i}$ and consider a set $S$ parameterized by $x_{i}=\left|S \cap X_{i}\right| /\left|X_{i}\right|$. The marginal value of $a$ added to $S$ is equal to

$$
\begin{aligned}
f_{S}(a) & =\tilde{f}\left(x_{1}, \ldots, x_{i}+\frac{1}{\left|X_{i}\right|}, \ldots, x_{k}\right)-\tilde{f}\left(x_{1}, \ldots, x_{i}, \ldots, x_{k}\right) \\
& =\int_{0}^{1 /\left|X_{i}\right|} \frac{\partial \tilde{f}}{\partial x_{i}}\left(x_{1}, \ldots, x_{i}+t, \ldots, x_{k}\right) d t .
\end{aligned}
$$

We want to prove that $f_{S}(a)$ cannot increase by adding elements to $S$, i.e. by increasing any coordinate $x_{j}$. Because $\frac{\partial \tilde{f}}{\partial x_{i}}$ is continuous and its derivative along $x_{j}, \frac{\partial^{2} \tilde{f}}{\partial x_{i} \partial x_{j}}$, is nonpositive except at finitely many points, $\frac{\partial \tilde{f}}{\partial x_{i}}$ is non-increasing in $x_{j}$. By shifting the entire integral to a higher value of $x_{j}$, the marginal value cannot increase.

Hence, we need our continuous functions to satisfy $\frac{\partial f}{\partial x_{i}} \geq 0$ and $\frac{\partial^{2} f}{\partial x_{i} \partial x_{j}} \leq 0$ for all $i, j \in[k]$, which implies monotonicity and submodularity in the discrete case. We call such functions smooth submodular. To shorten notation, we write $f(\mathbf{x})=f\left(x_{1}, \ldots, x_{k}\right)$.

In each instance, all players have the same valuation function. We find two functions $f, g$ such that we have $f(\mathbf{x})=$ $g(\mathbf{x})$ whenever $\max _{i, j}\left|x_{i}-x_{j}\right| \leq \epsilon$. As we show later, this will imply that $f$ and $g$ are indistinguishable by a subexponential number of queries. We construct these two functions as follows.

Lemma 2.4. For any $\beta>0$ and integer $k \geq 2$, there is $\epsilon>0$ and two smooth submodular functions $f, g:[0,1]^{k} \rightarrow$ $\mathbb{R}_{+}$such that

- If $\max _{i, j}\left|x_{i}-x_{j}\right| \leq \epsilon$, then $f(\mathbf{x})=g(\mathbf{x})$ and the function value depends only on $\bar{x}=\frac{1}{k} \sum_{i=1}^{k} x_{i}$.

- $\max \left\{\sum_{i=1}^{k} f\left(x_{i 1}, \ldots, x_{i k}\right) \mid x_{i j} \geq 0 \& \forall j ; \sum_{i=1}^{k} x_{i j}=\right.$ $1\} \geq(1-\beta) k$.

\footnotetext{
${ }^{1}$ To be more precise, on any axis-parallel line there are only finitely many points where $\frac{\partial^{2} \tilde{f}}{\partial x_{i} \partial x_{j}}$ is not defined.
}

- $\max \left\{\sum_{i=1}^{k} g\left(x_{i 1}, \ldots, x_{i k}\right) \mid x_{i j} \geq 0 \& \forall j ; \sum_{i=1}^{k} x_{i j}=\right.$ $1\} \leq\left(1-(1-1 / k)^{k}+\beta\right) k$.

Proof. We start by considering two smooth submodular functions, motivated by the examples of $k$-uniform hypergraphs that we discussed above.

- $f(\mathbf{x})=1-\prod_{i=1}^{k}\left(1-x_{i}\right)$.

- $g(\mathbf{x})=1-(1-\bar{x})^{k}$, where $\bar{x}=\frac{1}{k} \sum_{i=1}^{k} x_{i}$.

The optimal solution with valuation function $f$ is $x_{i i}=1$, $x_{i j}=0$ for $i \neq j$. This way, each player gets value 1 and $\sum_{i} f\left(x_{i 1}, \ldots, x_{i k}\right)=k$. For $g$, on the other hand, the value depends only on the average of the coordinates $\bar{x}$. By the concavity of $g$, the optimum solution is to set $x_{i j}=1 / k$ for all $i, j$, which gives total value $\sum_{i} g\left(x_{i 1}, \ldots, x_{i k}\right)=k(1-$ $\left.(1-1 / k)^{k}\right)$.

It remains to perturb the functions so that $f(\mathbf{x})=g(\mathbf{x})$ for vectors satisfying $\max _{i, j}\left|x_{i}-x_{j}\right| \leq \epsilon$. Let $h(\mathbf{x})$ denote the difference of the two functions,

$$
\text { - } h(\mathbf{x})=f(\mathbf{x})-g(\mathbf{x})=(1-\bar{x})^{k}-\prod_{i=1}^{k}\left(1-x_{i}\right) \text {. }
$$

Again, we denote $\bar{x}=\frac{1}{k} \sum_{i=1}^{k} x_{i}$. Also, let $\delta=\max _{i, j} \mid x_{i}-$ $x_{j} \mid$. First, we estimate $h(\mathbf{x})$ and its first derivatives in terms of $\bar{x}$ and $\delta$. We use very crude bounds, to simplify the analysis.

\section{Claim..}

1. $h(\mathbf{x}) \leq k \delta(1-\bar{x})^{k-1}$.

2. $h(\mathbf{x}) \geq k^{-4} \delta^{2}(1-\bar{x})^{k-2}$.

3. $\left|\frac{\partial h}{\partial x_{j}}\right| \leq k \delta(1-\bar{x})^{k-2}$, i.e. $\left|\frac{\partial h}{\partial x_{j}}\right| \leq k^{3}(1-\bar{x})^{k / 2-1} \sqrt{h(\mathbf{x})}$.

1.

We have $h(\mathbf{x})=(1-\bar{x})^{k}-\prod_{i=1}^{k}\left(1-x_{i}\right)$. If $k \delta \geq 1-\bar{x}$, we get immediately $h(\mathbf{x}) \leq(1-\bar{x})^{k} \leq k \delta(1-\bar{x})^{k-1}$. So let's assume $k \delta<1-\bar{x}$. Then, since $x_{i} \leq \bar{x}+\delta$ for all $i$, we get

$$
\begin{gathered}
h(\mathbf{x}) \leq(1-\bar{x})^{k}-(1-\bar{x}-\delta)^{k}= \\
(1-\bar{x})^{k}\left(1-\left(1-\frac{\delta}{1-\bar{x}}\right)^{k}\right) \leq(1-\bar{x})^{k} \frac{k \delta}{1-\bar{x}} .
\end{gathered}
$$

2.

For a lower bound on $h(\mathbf{x})$, suppose that $\delta=x_{2}-x_{1}$. For $k=2$, we are done immediately since

$$
h(\mathbf{x})=(1-\bar{x})^{2}-(1-\bar{x}-\delta / 2)(1-\bar{x}+\delta / 2)=\frac{1}{4} \delta^{2} .
$$

Hence, we assume $k>2$ and define $\eta=\frac{1}{k-2}\left(\bar{x}-\frac{1}{2}\left(x_{1}+\right.\right.$ $\left.x_{2}\right)$ ). I.e., $x_{1}=\bar{x}-(k-2) \eta-\delta / 2, x_{2}=\bar{x}-(k-2) \eta+\delta / 2$, and the average of the remaining coordinates is $\bar{x}+2 \eta$. By the arithmetic-geometric mean inequality, $\prod_{i \neq 1,2}\left(1-x_{i}\right)$ is maximized when these variables are all equal:

$$
\begin{aligned}
h(\mathbf{x}) \geq & (1-\bar{x})^{k}-(1-\bar{x}-2 \eta)^{k-2} \\
& \cdot\left(1-\bar{x}+(k-2) \eta-\frac{1}{2} \delta\right)\left(1-\bar{x}+(k-2) \eta+\frac{1}{2} \delta\right) \\
= & (1-\bar{x})^{k}-(1-\bar{x}-2 \eta)^{k-2}(1-\bar{x}+(k-2) \eta)^{2} \\
+ & +\frac{1}{4} \delta^{2}(1-\bar{x}-2 \eta)^{k-2} .
\end{aligned}
$$


Again by the arithmetic-geometric mean inequality, (1 $\bar{x})^{k} \geq(1-\bar{x}-2 \eta)^{k-2}(1-\bar{x}+(k-2) \eta)^{2}$. If $\eta \leq \frac{1}{k}(1-\bar{x})$, we are done because then the last term is at least $\frac{1}{4 e^{2}} \delta^{2}(1-\bar{x})^{k-2}$. So we can assume $\eta>\frac{1}{k}(1-\bar{x})$. In this case, we throw away the last term and write

$$
\begin{aligned}
& h(\mathbf{x}) \geq(1-\bar{x})^{k}-(1-\bar{x}+(k-2) \eta)^{2}(1-\bar{x}-2 \eta)^{k-2} \\
& =(1-\bar{x})^{k}\left(1-\left(1+(k-2) \frac{\eta}{1-\bar{x}}\right)^{2}\left(1-\frac{2 \eta}{1-\bar{x}}\right)^{k-2}\right) \\
& \geq(1-\bar{x})^{k}\left(1-\left(1+\frac{\eta}{1-\bar{x}}\right)^{2(k-2)}\left(1-\frac{\eta}{1-\bar{x}}\right)^{2(k-2)}\right) \\
& =(1-\bar{x})^{k}\left(1-\left(1-\frac{\eta^{2}}{(1-\bar{x})^{2}}\right)^{2(k-2)}\right) \\
& \geq(1-\bar{x})^{k}\left(1-\left(1-\frac{1}{k^{2}}\right)^{2(k-2)}\right) \geq \frac{1}{k^{2}}(1-\bar{x})^{k}
\end{aligned}
$$

using $k>2$ and $\eta>\frac{1}{k}(1-\bar{x})$. We observe it always holds that $\delta \leq k(1-\bar{x})$ : If the minimum coordinate is $x_{\text {min }}$, we have $\bar{x} \leq \frac{1}{k} x_{\min }+\frac{k-1}{k} \cdot 1$, hence $x_{\min } \geq k \bar{x}-(k-1)$ and $\delta \leq 1-x_{\min } \leq k(1-\bar{x})$.

Consequently, $h(\mathbf{x}) \geq k^{-2}(1-\bar{x})^{k} \geq k^{-4} \delta^{2}(1-\bar{x})^{k-2}$.

3.

Let $\delta=\max _{i, j}\left|x_{i}-x_{j}\right|$. We estimate the partial derivative

$$
\frac{\partial h}{\partial x_{j}}=\prod_{i \neq j}\left(1-x_{i}\right)-(1-\bar{x})^{k-1} .
$$

Define $\eta=\frac{1}{k-1}\left(x_{j}-\bar{x}\right)$. I.e., $x_{j}=\bar{x}+(k-1) \eta$ and the average of the remaining coordinates is $\bar{x}-\eta$. By the arithmeticgeometric mean inequality,

$$
\begin{aligned}
& \frac{\partial h}{\partial x_{j}} \leq(1-\bar{x}+\eta)^{k-1}-(1-\bar{x})^{k-1} \\
= & (1-\bar{x})^{k-1}\left(\left(\left(1+\frac{\eta}{1-\bar{x}}\right)^{k-1}-1\right) .\right.
\end{aligned}
$$

Since $\eta=\frac{1}{k-1}\left(x_{j}-\bar{x}\right) \leq \frac{1}{k-1}(1-\bar{x})$, we can estimate $(1+$ $\left.\frac{\eta}{1-\bar{x}}\right)^{k-1} \leq 1+2 k \frac{\eta}{1-\bar{x}}$. Also, we know that all coordinates differ from $\bar{x}-\eta$ by at most $\delta$, in particular $x_{j}=\bar{x}+(k-$ 1) $\eta \leq \bar{x}-\eta+\delta$, hence $k \eta \leq \delta$ and

$$
\frac{\partial h}{\partial x_{j}} \leq(1-\bar{x})^{k-1} \cdot 2 k \frac{\eta}{1-\bar{x}} \leq 2 \delta(1-\bar{x})^{k-2} .
$$

For a lower bound, it's enough to observe that each coordinate is at most $\bar{x}+\delta$, and so

$$
\begin{aligned}
\frac{\partial h}{\partial x_{j}} & \geq(1-\bar{x}-\delta)^{k-1}-(1-\bar{x})^{k-1} \\
& =(1-\bar{x})^{k-1}\left(\left(1-\frac{\delta}{1-\bar{x}}\right)^{k-1}-1\right) \\
& \geq(1-\bar{x})^{k-1}\left(-(k-1) \frac{\delta}{1-\bar{x}}\right) \\
& =-(k-1) \delta(1-\bar{x})^{k-2}
\end{aligned}
$$

assuming that $(k-1) \frac{\delta}{1-\bar{x}} \leq 1$; otherwise we get the same bound directly from $\frac{\partial h}{\partial x_{j}} \geq-(1-\bar{x})^{k-1}$. This finishes the proof of the claim.

We return to our construction. We define $\tilde{f}(\mathbf{x})=f(\mathbf{x})-$ $\phi(h(\mathbf{x}))$ where $\phi: \mathbb{R} \rightarrow \mathbb{R}$ is defined so that $\phi(t)=t$ for small $t \geq 0$, then $\phi(t)$ is increasing and concave with a controlled second derivative and finally $\phi(t)$ is bounded by a small constant everywhere. More precisely,

- For $t \in\left[0, \epsilon_{1}\right]$, we set $\phi(t)=t$. We choose $\epsilon_{1}=k \epsilon$. I.e., for $\max _{i, j}\left|x_{i}-x_{j}\right| \leq \epsilon$, we have $h(\mathbf{x}) \leq \epsilon_{1}$ by Claim 1 and then $\tilde{f}(\mathbf{x})=g(\mathbf{x})$.

- For $t \in\left[\epsilon_{1}, \epsilon_{2}\right]$, the first derivative of $\phi$ is continuous at $t=\epsilon_{1}$ and its second derivative is $\phi^{\prime \prime}(t)=-\alpha / t$ for $t \in\left[\epsilon_{1}, \epsilon_{2}\right]$. Hence,

$$
\phi^{\prime}(t)=1-\int_{\epsilon_{1}}^{t} \frac{\alpha}{\tau} d \tau=1-\alpha \ln \frac{t}{\epsilon_{1}} .
$$

We choose $\alpha=2 / \ln \frac{1}{\epsilon_{1}}$ and $\epsilon_{2}=\sqrt{\epsilon_{1}}$, so that $\phi^{\prime}\left(\epsilon_{2}\right)=$ 0 . Since $0 \leq \phi^{\prime}(t) \leq 1$ everywhere, we have $0 \leq$ $\phi\left(\epsilon_{2}\right) \leq \epsilon_{2}$.

- For $t>\epsilon_{2}$, we set $\phi(t)=\phi\left(\epsilon_{2}\right)$.

Hence, we have $0 \leq \phi(t) \leq \epsilon_{2}$ everywhere and $\tilde{f}(\mathbf{x})=f(\mathbf{x})-$ $\phi(h(\mathbf{x})) \geq f(\mathbf{x})-\epsilon_{2}$. Next, we want to show that we didn't corrupt the monotonicity and submodularity of $f$ too badly. We have

$$
\frac{\partial \tilde{f}}{\partial x_{j}}=\frac{\partial f}{\partial x_{j}}-\phi^{\prime}(h) \frac{\partial h}{\partial x_{j}}=\left(1-\phi^{\prime}(h)\right) \frac{\partial f}{\partial x_{j}}+\phi^{\prime}(h) \frac{\partial g}{\partial x_{j}} .
$$

We have $0 \leq \phi^{\prime}(h) \leq 1$, and $\frac{\partial f}{\partial x_{j}}$, $\frac{\partial g}{\partial x_{j}}$ are both nonnegative. So, $\frac{\partial \tilde{f}}{\partial x_{j}} \geq 0$. For the second partial derivatives, we get

$$
\begin{aligned}
\frac{\partial^{2} \tilde{f}}{\partial x_{i} \partial x_{j}} & =\frac{\partial^{2} f}{\partial x_{i} \partial x_{j}}-\phi^{\prime}(h) \frac{\partial^{2} h}{\partial x_{i} \partial x_{j}}-\phi^{\prime \prime}(h) \frac{\partial h}{\partial x_{i}} \frac{\partial h}{\partial x_{j}} \\
= & \left(1-\phi^{\prime}(h)\right) \frac{\partial^{2} f}{\partial x_{i} \partial x_{j}}+\phi^{\prime}(h) \frac{\partial^{2} g}{\partial x_{i} \partial x_{j}}-\phi^{\prime \prime}(h) \frac{\partial h}{\partial x_{i}} \frac{\partial h}{\partial x_{j}} .
\end{aligned}
$$

The first two terms form a convex combination of non-positive values. To control the third term, we have $\left|\phi^{\prime \prime}(h)\right| \leq \alpha / h$. In Claim 3, we showed that

$$
\left|\frac{\partial h}{\partial x_{i}}\right| \leq k^{3}(1-\bar{x})^{k / 2-1} \sqrt{h(\mathbf{x})} .
$$

We can conclude that

$$
\frac{\partial^{2} \tilde{f}}{\partial x_{i} \partial x_{j}} \leq\left|\phi^{\prime \prime}(h) \frac{\partial h}{\partial x_{i}} \frac{\partial h}{\partial x_{j}}\right| \leq \alpha k^{6}(1-\bar{x})^{k-2} .
$$

We need to make the second partial derivatives non-positive. Since $\frac{\partial^{2} g}{\partial x_{i} \partial x_{j}}=-\frac{k-1}{k}(1-\bar{x})^{k-2}$, it is enough to add a suitable multiple of $g$ to both functions: $\hat{f}=\tilde{f}+2 \alpha k^{6} g$, $\hat{g}=\left(1+2 \alpha k^{6}\right) g$. Then $\hat{f}, \hat{g}$ are smooth submodular.

Recall that we have $\alpha=2 / \ln \frac{1}{k \epsilon}$. For a given $\beta>0$, we choose $\epsilon=\frac{1}{k} e^{-4 k^{6} / \beta}$, so that $\beta=2 \alpha k^{6}$ and we increase $g$ only by a factor of $1+\beta$. We also get $\epsilon_{2}=\sqrt{k \epsilon} \leq \beta$, and therefore $\hat{f}(\mathbf{x}) \geq \tilde{f}(\mathbf{x}) \geq f(\mathbf{x})-\epsilon_{2} \geq f(\mathbf{x})-\beta$. Thus $\hat{f}$ and $\hat{g}$ satisfy the conditions of the lemma.

Now we are ready to prove Theorem 2.1.

Proof. Consider a large set of elements $X$, partitioned into equal parts $X_{1}, \ldots, X_{k}$. Lemma 2.4 defines two smooth submodular functions. By Lemma 2.3, we define discrete utility functions $f, g: 2^{X} \rightarrow \mathbb{R}_{+}$. We consider two instances, where all utility functions are equal to either $f$ or $g$. We 
present one of these two instances to a (possibly randomized) algorithm. The labeling of the elements of $X$ is arbitrary and unknown to the algorithm; we can assume that it is uniformly random.

Let us assume that an algorithm queries a set $S$ of size $s$. Let $x_{i}=\left|S \cap X_{i}\right| /\left|X_{i}\right|$. Since the partition is uniformly random, each $x_{i}$ is a random variable of expectation $s / k$ and variance $O(s)$. We consider $k$ fixed here, while the number of elements $m=|X|$ is very large. We can assume that $s \geq$ $\epsilon m$, otherwise the deviation of $x_{i}$ from its expectation can never be more than $\epsilon m$. Otherwise, by standard bounds, the probability of $x_{i}$ deviating from its expectation by more than $\epsilon m$ decays as $e^{-\Omega\left(\epsilon^{2} m^{2} / s\right)}=e^{-\Omega\left(\epsilon^{2} m\right)}$. Hence, it happens only with exponentially small probability that $\left|x_{i}-x_{j}\right|>\epsilon$ for any $i \neq j$. Let's call such a query unbalanced.

Consider any fixed sequence of $q$ queries. Unless the number of queries $q$ is exponentially large in $m$, it still happens only with an exponentially small probability that any query is unbalanced (by the union bound). Therefore, with high probability, no query is unbalanced.

Now consider any (possibly randomized) algorithm, using a subexponential number of value queries. In the randomized case, let us condition on the random bits on the algorithm. Given this, the sequence of queries can depend only on the obtained answers. Note that for balanced queries, we have $f(\mathbf{x})=g(\mathbf{x})$ and the function value depends only on $\bar{x}=\frac{1}{k} \sum_{i=1}^{k} x_{i}$, i.e. on the size of the queried set, which is the algorithm's own choice. Hence with high probability, the algorithm always follows the same sequence of (balanced) queries and the answers obtained are the same for $f(\mathbf{x})$ and $g(\mathbf{x})$. Intuitively, the algorithm never learns any information about the partition $\left(X_{1}, X_{2}, \ldots, X_{k}\right)$.

In the case of a randomized algorithm, we can now average over the choices of its random bits. Still, with high probability it never asks any unbalanced query and cannot distinguish between $f(\mathbf{x})$ and $g(\mathbf{x})$. If the underlying instance corresponds to $f(\mathbf{x})$, the algorithm will never find any set whose value differs from that of $g(\mathbf{x})$, and hence any solution obtained is at most $\left(1-(1-1 / k)^{k}+\beta\right)$ of the optimum.

\section{VALUE-QUERY COMPLEXITY OF SUB- ADDITIVE WELFARE MAXIMIZATION}

In this section, we construct an example showing that it is impossible to achieve an approximation factor significantly better than $\frac{1}{\sqrt{m}}$ for the subadditive welfare problem, using a polynomial number of value queries.

In fact, we prove our result for the more restricted class of fractionally subadditive valuation functions $[12,6]$ that is known to strictly contain all submodular valuation functions. A fractionally subadditive function is the pointwise maximum over a set of linear valuation functions.

Definition 3.1. A linear valuation function (also known as additive) is a set function $a: 2^{M} \rightarrow \mathbb{R}_{+}$that assigns a non-negative value to every singleton $\{j \in M\}$, and for all $S \subseteq M$ it holds that $a(S)=\sum_{j \in S} a(\{j\})$.

DeFinition 3.2. A fractionally subadditive function is a set function $f: S^{M} \rightarrow \mathbb{R}_{+}$, for which there is a finite set of linear valuation functions $A=\left\{a_{1}, \ldots, a_{l}\right\}$ such that $f(S)=$ $\max _{a_{i} \in A} a_{i}(S)$, for every $S \subseteq M$.
We prove the following theorem.

TheOREm 3.1. For any fixed $\epsilon>0, a \frac{1}{m^{\frac{1}{2}-\epsilon}}$-approximation algorithm for fractionally subadditive valuation functions requires exponentially many value queries.

We note that the result of Theorem 3.1 can be shown to hold even for the case that bidders have the same valuation function (as will be explained later).

Proof. We shall use probabilistic arguments (similar to those in Section 2) to show that any algorithm that obtains an $\frac{1}{m^{\frac{1}{2}-\epsilon}}$-approximation to the social-welfare requires an exponential number of value queries. For simplicity, we shall start by proving the theorem for the case that bidders have different valuation functions. We shall later discuss how the proof can be extended to the more restricted case that all bidders have the same valuation function.

Fix a small constant $\delta>0$ (to be determined later). We shall construct a combinatorial auction with $m$ items and $k=\sqrt{m}$ bidders. For every $S$ let $a_{S}$ be the linear valuation function that assigns a value of 1 to each item $j \in S$, and 0 to each item $j \notin S$. Let $\bar{a}$ be the additive valuation that assigns every item $j \in[m]$ a value of $\frac{1+\delta}{m^{\frac{1}{2}-\delta}}$.

Let $v_{1}, \ldots, v_{k}$ be an $k$-tuple of (equal) valuation functions defined as follows:

$$
v_{i}=\max \left\{a_{S:|S| \leq(1+\delta) m^{2 \delta}}, \bar{a}\right\}
$$

That is, $v_{i}$ is the pointwise maximum over the set of additive valuation functions that contains $a_{S}$ for all $S$ of a certain size, and $\bar{a}$. Choose, uniformly at random, a partition of the items into $\sqrt{m}$ disjoint bundles of items $T_{1}, \ldots, T_{k}$ such that for each $i,\left|T_{i}\right|=\sqrt{m}$. Let $v_{1}^{\prime}, \ldots, v_{k}^{\prime}$ be the $k$-tuple of valuation functions defined as follows:

$$
v_{i}^{\prime}=\max \left\{v_{i}, a_{T_{i}}\right\}
$$

We shall prove that for every player $i$, it takes an exponential number of value queries to distinguish between the case that $i$ 's valuation function is $v_{i}$ and the case that $i$ 's valuation function is $v_{i}^{\prime}$. It is easy to see that the maximum social-welfare attainable if the valuation functions are $v_{1} \ldots, v_{k}$ is $O\left(m^{\frac{1}{2}+2 \delta}\right)$, while the optimal social-welfare if the valuation functions are $v_{1}^{\prime} \ldots, v_{k}^{\prime}$ is $m$. Hence, the fact that it requires an exponential number of value queries to distinguish between the valuation-functions profiles $v_{1} \ldots, v_{k}$ and $v_{1}^{\prime} \ldots, v_{k}^{\prime}$ implies that one cannot get an approximation ratio better than $\Omega\left(\frac{1}{m^{\frac{1}{2}-2 \delta}}\right)$ in less than an exponential number of value queries.

Consider a specific player $i$. Fix a bundle $S$ of size smaller or equal to $m^{\frac{1}{2}+\delta}$. It holds that $v_{i}(S)=\max \{|S|,(1+$ $\left.\delta) m^{2 \delta}\right\} . \quad v_{i}^{\prime}$ might assign a value higher than $v_{i}$ to $S$ but only if

$$
\left|S \cap T_{i}\right|>(1+\delta) m^{2 \delta} .
$$

Using standard probabilistic arguments, and relying on the Chernoff bounds, it can be shown that $\operatorname{Pr}\left[\left|S \cap T_{i}\right|>\right.$ $\left.(1+\delta) m^{2 \delta}\right]$ is exponentially small (see Section 2 ).

Now, consider a bundle $S$ of size greater than $m^{\frac{1}{2}+\delta} \cdot v_{i}$ will assign to $S$ the value of $(1+\delta) \frac{|S|}{m^{\frac{1}{2}-\delta}} \cdot v_{i}^{\prime}$ might assign $S$ a higher value, but only if

$$
\left|S \cap T_{i}\right|>(1+\delta) \frac{|S|}{m^{\frac{1}{2}-\delta}} .
$$


Again, using standard probabilistic arguments it can be shown that $\operatorname{Pr}\left[\left|S \cap T_{i}\right|>(1+\delta) \frac{|S|}{m^{\frac{1}{2}-\delta}}\right]$ is exponentially small.

We conclude that for every bundle $S$, only with exponentially small probability does one gather sufficient information to distinguish between the case that $i$ 's valuation is $v_{i}$ and the case that it is $v_{i}^{\prime}$. Hence, it requires an exponential number of value queries to distinguish between $v_{i}$ and $v_{i}^{\prime}$ in the worst case. This concludes the proof of the theorem.

We note that this proof can be extended to the case that all bidders have the same valuation function. Observe, that all the $v_{i}$ functions are identical. We shall show how it is possible to make all the $v_{i}^{\prime}$ identical as well. Informally, in the construction of $v_{i}^{\prime}$, we have associated every bidder $i$ with a bundle $T_{i}$. However, it is possible to define $v_{i}^{\prime}$ in a way that associates every bidder $i$ with the entire partition $T_{1}, \ldots, T_{k}$. This is done by defining each $v_{i}^{\prime}$ to be $\max \left\{v_{i}, a_{T_{1}}, \ldots, a_{T_{k}}\right\}$. It is still true that distinguishing between $v_{1}, \ldots, v_{k}$ and $v_{1}^{\prime}, \ldots, v_{k}^{\prime}$ requires an exponential number of value queries (using the same probabilistic arguments as before and the union bound). It is also still true that the ratio between the social welfare if the valuation functions are $v_{1}, \ldots, v_{k}$, and the social welfare if the valuation functions are $v_{1}^{\prime}, \ldots, v_{k}^{\prime}$ is $O\left(m^{\frac{1}{2}-2 \delta}\right)$ (the ratio between $O\left(m^{\frac{1}{2}+2 \delta}\right)$ and $m$, respectively). Hence, in order to obtain an approximationratio better than $\Omega\left(\frac{1}{m^{\frac{1}{2}-2 \delta}}\right)$, an exponential number of value queries in required.

\section{VALUE-QUERY COMPLEXITY OF SU- PERADDITIVE WELFARE MAXIMIZA- TION}

In this section, we construct an example showing that it is impossible to achieve an approximation factor significantly better than $\frac{\log m}{m}$ for the superadditive welfare problem, using a polynomial number of value queries.

The construction of the example will be done in two steps. First, we shall define a subclass of superadditive valuation functions we term min-linear functions. This is a superadditive analogue of fractionally subadditive functions $[12,6]$. We shall then prove our lower bound for this more restricted class.

DeFinition 4.1. A min-linear function is a set function $f: 2^{M} \rightarrow \mathbb{R}_{+}$such that there is a finite set of linear valuation functions $A=\left\{a_{1}, \ldots, a_{l}\right\}$ such that for every $S \subseteq M$ $f(S)=\min _{a_{i} \in A}\left\{a_{i}(S)\right\}$.

It is easy to show (and analogous to the proofs in $[12,11]$ ) that min-linear functions are contained in the class of superadditive valuation functions, and are a superclass of supermodular valuation functions. For completeness we present the simple proofs below. Simple examples demonstrate that these containments are strict.

\section{Claim 4.1. Any min-linear function is superadditive.}

Proof. Let $f=\min _{a \in A} a$ be a min-linear function. Let $S$ and $T$ be two disjoint subsets of items. By definition there are linear functions $a_{S}, a_{T}$, and $a_{S \cup T}$ in $A$ for which the value of $S, T$, and $S \cup T$, is minimized. Therefore, it must hold that $a_{S}(S) \leq a_{S \cup T}(S)$ and $a_{T}(T) \leq a_{S \cup T}(T)$.
Hence:

$$
\begin{aligned}
f(S \cup T) & =a_{S \cup T}(S \cup T) \\
& =a_{S \cup T}(S)+a_{S \cup T}(T) \\
& \geq a_{S}(S)+a_{T}(T) \\
& =f(S)+f(T) .
\end{aligned}
$$

DeFinition 4.2. A set function $f$ is supermodular iff for any two sets $S$ and $T, f(S)+f(T) \leq f(S \cup T)+f(S \cap T)$.

Claim 4.2. Any supermodular valuation function is a minlinear function.

Proof. Let $f$ be a supermodular valuation function. Fix an order on the items, w.l.o.g., $1, \ldots, m$. For every set $S$ we define a linear function $a_{S}$ as follows: For every $j \in S$ $a_{S}(\{j\})=f(\{1, \ldots, j\})-f(\{1, \ldots, j-1\})$. For every $j \notin S$ $a_{S}(\{j\})=\infty$, where $\infty$ represents a very large number (in particular $f(M)<<\infty)$. It is easy to see that $a_{S}(S)=f(S)$ for any $S$. We want to show that $f$ is min-linear and the finite set of linear functions is $A=\left\{a_{T}\right\}_{T \subseteq M}$. For this, we need to show that $a_{S}(S)=\min _{a_{T} \in A} a_{T}(S)$.

Observe that for any $S, T$ such that $S$ is not contained in $T$, it is impossible that the minimum for $S$ is achieved by $a_{T}$ (because for some $j \in S a_{T}(\{j\})=\infty$ ). So, we are left with the case that $S \subseteq T$. Here we exploit the well-known fact that monotone supermodular functions have increasing marginal values. That is, if $U \subset V \subseteq M$, and $j$ is in neither $U$ nor $V$, then $f(U \cup\{j\})-f(U) \leq f(V \cup\{j\})-f(V)$. It is easy to see that this implies that for $S \subseteq T$, for any $j \in S a_{S}(\{j\}) \leq a_{T}(\{j\})$. Hence, $a_{S}$ is indeed the linear function for which the minimum is achieved (for bundle $S$ ). This implies that $f=\min A$.

We are now ready to prove the following theorem:

THEOREM 4.1. For any $\epsilon>0, a \frac{\log ^{1+\epsilon} m}{m}$-approximation algorithm for min-linear valuation functions requires a superpolynomial number of value queries.

We note that the result of Theorem 4.1 can be shown to hold even for the case that all bidders have the same valuation function (as will be discussed later).

Proof. For simplicity, we shall start by proving the theorem for the case that bidders have different valuation functions. We shall later discuss how the proof can be extended to the more restricted case that all bidders have the same valuation function.

We use probabilistic arguments similar to those in Section 2. Fix $\epsilon>0$. We construct an auction with $|M|=$ $m+\frac{m}{\log ^{1+\epsilon} m}$ items and $|N|=k=\frac{m}{\log ^{1+\epsilon} m} . \quad M$ consists of two disjoint sets $M_{1}$ and $M_{2}$, such that $\left|M_{1}\right|=m$ and $\left|M_{2}\right|=\frac{m}{\log ^{1+\epsilon} m}$. Each bidder $i \in[k]$ is associated with a unique item $d_{i}$ in $M_{2}$. We partition $M_{1}$, uniformly at random, into $k=\frac{m}{\log ^{1+\epsilon} m}$ bundles $T_{1}, \ldots, T_{k}$ of equal size (i.e., of size $\left.\log ^{1+\epsilon} m\right)$.

For each bidder $i$, let $b_{i}$ be the linear valuation function that assigns a value of 1 to $d_{i}$ and 0 to all other items. By $a_{S}$, we denote a linear function that assigns a value of 1 to each item in $S$ and 0 to all other items.

Let $v_{1}, \ldots, v_{k}$ be the $k$-tuple of valuation functions defined as follows:

$$
v_{i}=\min \left\{b_{i}, a_{S: S \subseteq M_{1},|S|=\frac{m}{2}}\right\} .
$$


This function has a very simple interpretation: It assigns a value of 1 to every set that contains $d_{i}$ and more than $\frac{m}{2}$ items in $M_{1}$. It assigns 0 to all other bundles.

Let $v_{1}^{\prime}, \ldots, v_{k}^{\prime}$ be the $k$-tuple of valuation functions defined as follows:

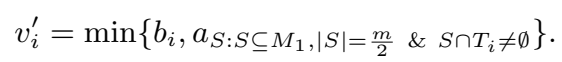

This function too has a simple interpretation: It assigns a value of 1 to every set that contains $d_{i}$ and $T_{i}$, or $d_{i}$ and more than $\frac{m}{2}$ items in $M_{1}$. It assigns 0 to all other sets.

We shall show that it would take a super-polynomial number of value queries to distinguish between the case that $i$ 's valuation function is $v_{i}$ and the case that it is $v_{i}^{\prime}$. It is easy to see that if the valuation functions of the bidders are $v_{1}, \ldots, v_{k}$ then the optimal social welfare is 1 . On the other hand, if the valuation functions of the players are $v_{1}^{\prime}, \ldots, v_{k}^{\prime}$ then the optimal social welfare value is $\frac{m}{\log ^{1+\epsilon} m}$ (assign every bidder $i$ the bundle that contains $d_{i}$ and $T_{i}$ ). Hence, it follows that achieving an approximation of $\frac{\log ^{1+\epsilon} m}{m}$ requires a super-polynomial number of value queries (required to distinguish between $v_{1}, \ldots, v_{k}$ and $v_{1}^{\prime}, \ldots, v_{k}^{\prime}$ ).

Observe that, for each $i, v_{i}$ and $v_{i}^{\prime}$ assign exactly the same value to all bundles, except for bundles that contain $d_{i}$ and at most $\frac{m}{2}$ items in $M_{1}$. Also observe, that the difference is that $v_{i}$ assigns a value of 0 to all these bundles, while $v_{i}^{\prime}$ assigns a value of 1 to such bundles that contain $T_{i}$ (and 0 to all other such sets). What is the probability that a set of size at most $\frac{m}{2}$ in $M_{1}$ contains $T_{i}$ ? Let $S$ be a bundle in $M_{1}$ of size at most $\frac{m}{2}$. Recall that $T_{i}$ is uniformly distributed over all sets of size $\log ^{1+\epsilon} m$. For every item in $T_{i}$, the probability that it is contained in $S$ is at most $\frac{1}{2}$. Therefore,

$$
\operatorname{Pr}\left[T_{i} \subseteq S\right] \leq \frac{1}{2^{\log 1+\epsilon} m}
$$

This implies that $2^{\log ^{1+\epsilon} m}$, i.e., a super-polynomial number of value queries, may be required to distinguish between $v_{i}$ and $v_{i}^{\prime}$. The theorem follows.

We note that this proof can be extended to the case that all bidders have the same valuation function. Observe that all the $v_{i}$ functions are identical. So, it suffices to show that the $v_{i}^{\prime}$ s can be converted to be identical, while still maintaining the following properties: It must require a superpolynomial number of value queries to distinguish between $v_{1}, \ldots, v_{k}$ and $v_{1}^{\prime}, \ldots, v_{k}^{\prime}$. The ratio between the social welfare if the valuation functions are $v_{1}, \ldots, v_{k}$, and the social welfare if the valuation functions are $v_{1}^{\prime}, \ldots, v_{k}^{\prime}$ must remain $\frac{m}{\log ^{1+\epsilon} m}$ (the ratio between 1 and $\frac{m}{\log ^{1+\epsilon} m}$, respectively). This can be done in a way analogous to that shown in the proof of Theorem 3.1. Informally, in the construction of $v_{i}^{\prime}$, we have associated every bidder $i$ with a bundle $T_{i}$. However, it is possible to define $v_{i}^{\prime}$ in a way that associates every bidder $i$ with the entire partition $T_{1}, \ldots, T_{k}$.

\section{OPEN QUESTIONS}

We conclude by exhibiting the following two open questions:

- For the case of submodular valuation functions, the only information-theoretic lower bound in the models of general and demand queries is $1-O(1 / m)$. There are indications that suggest the existence of a constant $(1-\epsilon)$ lower bound (APX-hardness results in the demand query and general query models $[3,7]$, and an integrality gap [4]). Proving such a lower bound in any of these two models is a very interesting open question and seems to require non-trivial combinatorial constructions.

- We have presented tight lower bounds in the value query model for submodular and subadditive valuation functions. There is still a gap between $\frac{\sqrt{\log m}}{m}$ and $\frac{\log m}{m}$ in the case of superadditive valuations. We have not considered the class of supermodular valuation functions, for which no information-theoretic lower bound is known in any of the models. Proving informationtheoretic lower bounds for this class is an open problem.

\section{REFERENCES}

[1] L. Blumrosen and N. Nisan. On the computational power of iterative auctions I: Demand queries. In Proc. of the 6th ACM Conference on Electronic Commerce (EC), 2005.

[2] L. Blumrosen and N. Nisan. On the computational power of iterative auctions I: Ascending auctions. In Proc. of the 6th ACM Conference on Electronic Commerce (EC), 2005.

[3] S. Dobzinski. Private communication, 2006.

[4] S. Dobzinski and M. Schapira. An improved approximation algorithm for combinatorial auctions with submodular bidders. In Proc. of the 18rd Annual ACM Symposium on Discrete Algorithms (SODA), 2006, 1064-1073.

[5] S. Dobzinski, N. Nisan and M. Schapira. Approximation algorithms for combinatorial auctions with complement-free bidders. In Proc. of the 37th Annual ACM Symposium on Theory of Computing (STOC), 2005, 610-618.

[6] U. Feige. On maximizing welfare when utility functions are subadditive. In Proc. of the 38th Annual ACM Symposium on Theory of Computing (STOC), 2006, 41-50.

[7] U. Feige and J. Vondrák. Approximation algorithms for combinatorial allocation problems: Improving the factor of $1-1 / e$. In Proc. of the 47th Annual IEEE Symposium on the Foundations of Computer Science (FOCS), 2006, 667-676.

[8] U. Feige, V. Mirrokni and J. Vondrák. Maximizing non-monotone submodular functions. In Proc. of the 47th Annual IEEE Symposium on the Foundations of Computer Science (FOCS), 2007, 461-471.

[9] R. Holzman, N. Kfir-Dahav, D. Monderer and M. Tennenholtz. Bundling equilibrium in combinatorial auctions. Games and Economic Behavior 47, 2004, 104-123.

[10] S. Khot, R. Lipton, E. Markakis and A. Mehta. Inapproximability results for combinatorial auctions with submodular utility functions. In Proc. of WINE 2005.

[11] B. Lehmann, D. Lehmann and N. Nisan. Combinatorial auctions with decreasing marginal utilities In Proc. of the 3rd ACM Conference on Electronic Commerce (EC), 2001. 
[12] N. Nisan. Bidding and allocation in combinatorial auctions. In Proc. of the 2nd ACM Conference on Electronic Commerce (EC), 2000.

[13] N. Nisan and I. Segal. The communication requirements of efficient allocations and supporting prices. Journal of Economic Theory, 129:1, 2006, $192-224$.
[14] G. Nemhauser and M. Fisher, and L. Wolsey. An analysis of approximations for maximizing submodular set functions II. Math. Programming Study 8, 1978, $73-87$.

[15] J. Vondrák. Optimal approximation for the Submodular Welfare Problem in the value oracle model. To appear in Proc. of the 40th Annual ACM Symposium on Theory of Computing (STOC), 2008. 